\title{
Indicación farmacéutica para el dolor lumbar en pacientes con diabetes y/o hipertensión arterial que acuden a la farmacia comunitaria
}

\author{
Jesús López-Torres López ${ }^{1}$, José Antonio Carbajal de Lara ${ }^{1,2}, M^{a}$ Rosa López-Torres Hidalgo ${ }^{1,2}$ \\ 1. Farmacéutico comunitario. Albacete. 2. Profesor asociado. Facultad de Farmacia. Universidad de Castilla-La Mancha.
}

\section{PALABRAS CLAVE}

Dolor lumbar, revisión, indicación farmacéutica farmacia comunitaria, algoritmo de actuación

\section{ABREVIATURAS}

AINE: antiinflamatorios no esteroideos

ARA II: antagonistas de los receptores de la angiotensina II BOT plus 2.0: Base de Datos del Conocimiento Sanitario del Consejo General de Colegios Oficiales de Farmacéuticos

CMA: Canadian Medical Association

COXIB: inhibidor selectivo de la ciclooxigensa-2 DM: diabetes mellitus GPC: guías de práctica clínica GRADE: Grading of Recommendations Assessment, Development and Evaluation HTA: hipertensión arterial IECA: inhibidor de la enzima conversora de la angiotensina NHS: National Health Service PRISMA: Preferred Reporting Items for Systematic review and Meta-Analysis Guidelines Network

\section{KEYWORDS}

Low-back pain, review, pharmaceutical counselling, community pharmacy, performance algorithm

Recibido: 15/10/2016 Aceptado: 29/11/2016 Disponible online: $30-12-2016$ SIGN: Scottish Intercollegiate

\section{RESUMEN}

El dolor lumbar constituye un motivo muy frecuente de consulta en la farmacia, por lo que la indicación farmacéutica debe estar basada en criterios científicos que contribuyan a resolverlo, sin que el control de las cifras de presión arterial o de glucemia pueda verse alterado en el caso de pacientes con hipertensión arterial o diabetes.

Mediante una revisión sistemática de Guías de Práctica Clínica (GPC) dirigidas al abordaje del dolor lumbar, así como de otras fuentes bibliográficas, se han elaborado recomendaciones sobre indicación farmacéutica a pacientes con diabetes y/o hipertensión arterial que acuden a la oficina de farmacia solicitando tratamiento farmacológico para dicho síntoma.

Tras diseñar una estrategia de búsqueda, se realizó la selección de las fuentes bibliográficas y se llevó a cabo la recogida de información. La búsqueda bibliográfica se realizó en los siguientes recursos web de almacenamiento o búsqueda de GPC: Medline, GuíaSalud, National Guideline Clearinghouse, CMA Infobase, Scottish Intercollegiate Guidelines Network, Australia's Clinical Practice Guidelines Portal, Trip Database y National Health Service Evidence. Como fuentes complementarias, se consultó UpToDate, Base de Datos B0T plus 2.0 y un tratado de Farmacología Humana.

Las recomendaciones específicas sobre tratamiento farmacológico fueron formuladas teniendo en cuenta su nivel de evidencia y se revisaron las posibles interacciones y/o contraindicaciones de los fármacos en los pacientes con hipertensión arterial y/o diabetes mellitus. Así mismo, se construyó un algoritmo de actuación, siguiendo las Buenas Prácticas en Farmacia Comunitaria en España elaboradas por el Consejo General de Colegios Oficiales de Farmacéuticos.

Las recomendaciones se refieren al manejo del dolor lumbar inespecífico e incluyen por lo general, como tratamiento de primera línea por su mejor perfil riesgo/beneficio, el uso de paracetamol en el dolor lumbar agudo o subagudo de forma aislada y de origen musculoesquelético, sin sobrepasar 3 ó 4 g/día

\section{Pharmaceutical counselling for back pain in patients with diabetes or hypertension in community pharmacy}

\section{ABSTRACT}

Lumbar pain is a frequent reason for consultation in the pharmacy, so the pharmaceutical indication must be based on scientific criteria that contribute to solve it, without the control of the blood pressure or blood sugar figures can be altered in the case of patients with hypertension or diabetes.

Through a systematic review of Clinical Practice Guidelines (CPG) aimed at addressing back pain, as well as other literature sources, recommendations have been made on minor ailment scheme for patients with diabetes and/or hypertension who come to the office of Pharmacy requesting pharmacological treatment for said symptom.

After designing a search strategy, the selection of the bibliographic sources was carried out and the collection of information was carried out. The literature search was conducted in the following web storage resources or GPC search: Medline, GuíaSalud, National Guideline Clearinghouse, CMA Infobase, Scottish Intercollegiate Guidelines Network, Australia’s Clinical Practice Guidelines Portal, Trip Database and National Health Service Evidence. As additional sources, were consulted of UpToDate, Database BOT plus 2.0 and a treaty of Human Pharmacology.

Specific recommendations on drug treatment were formulated taking into account their level of evidence and possible interactions and contraindications of drugs in patients with hypertension and/or diabetes mellitus. Likewise, performance algorithm were built, following Good Practices in Community Pharmacy in Spain of General Council of Official Colleges of Pharmacists.

The recommendations refer to the management of non-specific low-back pain and generally include, as a first-line treatment due to its better risk / benefit profile, the use of paracetamol in acute or subacute low back pain in isolation and of musculoskeletal origin, without exceeding 3 or $4 \mathrm{~g} /$ day.

Financiación: Ninguna ajena.

Conflicto de intereses: Los autores declaran no existir conflicto de intereses en relación con el contenido del articulo.

Contribución a la autoría: JL-TL Participó en el diseño del estudio, el desarrollo de la investigación, el análisis de los resultados y su tratamiento estadistico, en la redacción del manuscrito y en la revisión del contenido final. JACL y MRL-TH en el diseño del estudio, el desarrollo de la investigación, la redacción del manuscrito y en la revisión del contenido final.

Cite este artículo como: López-Torres J, Carbajal JA, López-Torres MR. Indicación farmacéutica para el dolor lumbar en pacientes con diabetes y/o hipertensión arterial que acuden a la farmacia comunitaria. Farmacéuticos Comunitarios. 2016 Dec 30; 8(4):34-41. doi:10.5672/FC.2173-9218. (2016/Nol8).004.05

Autor para correspondencia: Jesús López-Torres López (jlopeztl@yahoo.es) 


\section{Introducción}

La contribución de los profesionales farmacéuticos es esencial en el proceso de atención a la salud. Con frecuencia, cuando las personas perciben sus problemas de salud como banales acuden al farmacéutico para que les ayude a solucionarlos. Los sintomas menores se entienden como problemas de salud de carácter no grave, autolimitados o de corta duración, que no tienen relación alguna con las manifestaciones clínicas de los otros problemas de salud que sufra el paciente, ni con los efectos, deseados o no, de los medicamentos que toma, que no precisa por tanto de diagnóstico médico y que responden o se alivian con un tratamiento sintomático, o aquellos problemas de salud para los que el farmacéutico esté autorizado [1]. Al respecto, el abordaje de los síntomas menores exige que la actuación de dicho profesional esté orientada por criterios científicos, basados en la mejor evidencia disponible, para así mejorar la calidad de la atención y aplicar los hallazgos de la investigación en la toma de decisiones.

La actuación del farmacéutico comunitario, ante un paciente con síntomas o trastornos banales que acude a la farmacia buscando la asesoría o el consejo de este profesional, se ha definido como "indicación farmacéutica”, la cual se concibe como el acto profesional por el que el farmacéutico se responsabiliza de la selección de un medicamento que no necesita receta médica, con el objetivo de aliviar o resolver un problema de salud a instancias del paciente, o bien su derivación al médico cuando dicho problema necesite de su actuación [2]. En este contexto, los objetivos del farmacéutico incluyen la valoración de la severidad de los síntomas, la elección de la opción más adecuada para resolver el problema de salud, incluyendo la derivación al médico si es oportuna, la indicación de un medicamento sin receta médica, o bien de medidas no farmacológicas, y la información al paciente acerca de posibles efectos adversos de la medicación indicada.

La actuación del farmacéutico requiere la realización de una breve entrevista con el paciente para recoger información sobre el motivo de consulta, la duración de los sintomas y la presencia de otros síntomas de alar- ma que aconsejen la valoración del médico [3,4]. A su vez, la información proporcionada al paciente requiere de conocimientos actualizados sobre los principios activos disponibles sin receta, especialmente sobre su utilidad y su seguridad en el tratamiento de los síntomas menores.

Con frecuencia, las personas que solicitan información en la farmacia para resolver sus síntomas menores presentan enfermedades crónicas y también con frecuencia se trata de pacientes polimedicados. Entre las comorbilidades más frecuentes se encuentran la hipertensión arterial (HTA) y la diabetes mellitus (DM). La HTA presenta una prevalencia considerable en los países desarrollados y afecta en España al 35\% de los adultos, llegando a un $40 \%$ en edades medias y al 68\% en mayores de 60 años [5]. En cuanto a la DM, constituye una de las enfermedades con mayor impacto sociosanitario en España, cuya prevalencia estimada es del $6,2 \%$ para el intervalo de edad de 30-65 años y del 10\% para el de 30-89 años [6]. En ambos tipos de pacientes la indicación farmacéutica debe contemplar específicamente tanto la efectividad como la seguridad en el tratamiento de los síntomas menores. Además de la selección de los medicamentos más adecuados para estos pacientes, el farmacéutico debe proporcionar una información mínima sobre el objetivo del tratamiento, la pauta, la posología, la duración del mismo y qué hacer en caso de no mejoría o empeoramiento.

Además de contribuir al abordaje de los síntomas menores en los pacientes hipertensos, los farmacéuticos comunitarios, teniendo en cuenta su accesibilidad y experiencia en la terapia con medicamentos, son una opción lógica y un activo muy valioso para mejorar el manejo de la HTA [7], como ha sido comprobado en algunas revisiones sistemáticas de ensayos controlados aleatorios [8] dirigidos a evaluar el efecto de las intervenciones del farmacéutico. Así, el cuidado farmacéutico es capaz de reducir significativamente tanto la presión sistólica como diastólica y, como consecuencia, el riesgo de complicaciones cardiovasculares.

De igual forma, los resultados de estudios previos [9] muestran cómo el consejo farmacéutico contribuye a mejorar el control metabólico en pacientes con diabetes tipo 2. En un metaanálisis [10] llevado a cabo en 15 ensayos aleatorios, incluyendo un total de 9.111 pacientes, se comprobó que las intervenciones del profesional farmacéutico permiten reducir eficazmente el riesgo cardiovascular, a través de un mejor control de la presión arterial, colesterol total e índice de masa corporal.

El dolor lumbar [11] constituye un motivo muy frecuente de consulta en la farmacia, por lo que la indicación farmacéutica debe estar basada en criterios científicos que contribuyan a resolverlo, sin que se aumente el riesgo cardiovascular y teniendo en cuenta si se trata de personas con o sin eventos cardiovasculares previos. Consiste en molestias en el último tramo de la columna vertebral que pueden ir acompañadas de limitación de la movilidad. El dolor de espalda es una patología que afecta prácticamente a toda la población en algún momento de la vida.

Por otra parte, debe vigilarse que la indicación farmacéutica no interfiera con la medicación habitual de dichos pacientes, con frecuencia polimedicados. Esta circunstancia facilita la aparición de interacciones cuyo resultado puede ser una reacción adversa o la pérdida de efecto terapéutico. $\mathrm{Su}$ incidencia es difícil de determinar, pero se relaciona fundamentalmente con el número de fármacos administrados conjuntamente al mismo paciente. Alrededor del 10\% de las reacciones adversas a medicamentos y un porcentaje desconocido, pero probablemente elevado, de los fracasos terapéuticos se deben a interacciones que en muchas ocasiones son evitables [12]. Conocer su existencia y mecanismos de producción ayuda a identificarlas y prevenirlas.

El objetivo ha consistido en formular recomendaciones de indicación farmacéutica a pacientes adultos con diabetes y/o hipertensión arterial que acuden a la farmacia comunitaria solicitando tratamiento farmacológico para el dolor lumbar.

\section{Materiales y métodos}

Antes de elaborar las recomendaciones se llevó a cabo una revisión sistemática de las guías de práctica clínica (GPC) dirigidas al abordaje del dolor lumbar, así como de otras 
Guías de Práctica Clínica y otros documentos pertinentes identificados mediante una búsqueda sistemática

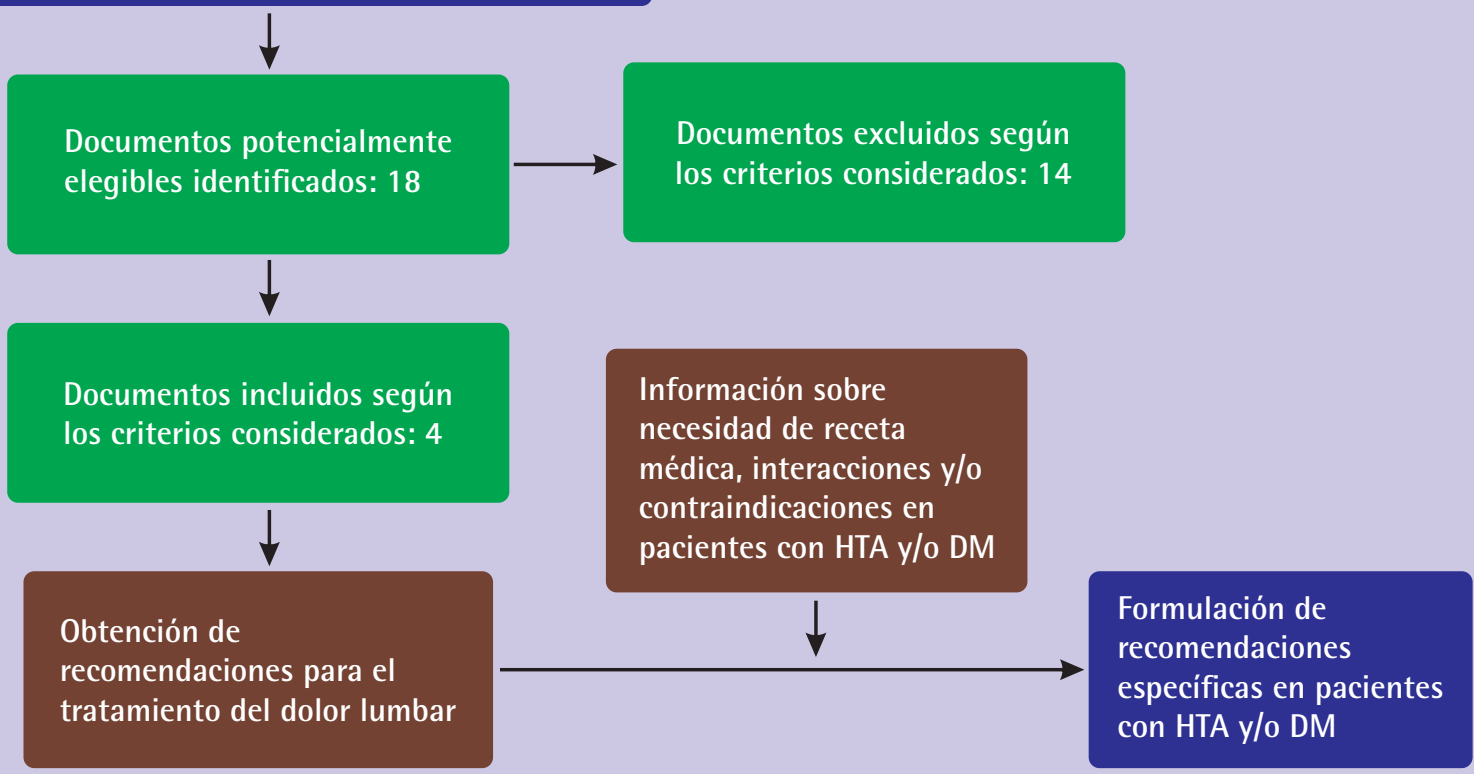

Figura 1 Esquema general del estudio

fuentes bibliográficas, siguiendo la metodología propuesta en la declaración PRISMA [13], la cual se dirige a mejorar la publicación de revisiones sistemáticas. Tras diseñar la estrategia de búsqueda, se realizó la selección de dichas fuentes bibliográficas y se llevó a cabo la recogida de información (figura 1).

La búsqueda bibliográfica, utilizando como palabras clave "lumbalgia" o "low back pain", se realizó en los siguientes recursos web de almacenamiento o búsqueda de GPC: Medline vía Pubmed [14], GuíaSalud [15] (Biblioteca de Guías de Práctica Clínica del Sistema Nacional de Salud), National Guideline Clearinghouse [16], Canadian Medical Association (CMA) Infobase [17], Scottish Intercollegiate Guidelines Network [18] (SIGN), Australia's Clinical Practice Guidelines Portal [19], Trip Database [20] y National Health Service (NHS) Evidence [21]. Como fuente complementaria se consultó UpToDate [22], que constituye un recurso de ayuda para la toma de decisiones clínicas basado en evidencia.

Para realizar la búsqueda en Medline, vía Pubmed, se consideraron los siguientes términos: Low back pain [Title/Abstract] AND (Practice Guideline [ptyp] AND “2006/03/23” [PDat]:
“2016/03/19" [PDat] AND "humans" [MeSH Terms] AND "adult" [MeSH Terms]).

Los criterios que se utilizaron para la selección de las GPC fueron los siguientes: 1) GPC directamente relacionadas con el tratamiento farmacológico del dolor lumbar; 2) GPC basadas en la evidencia.

Como criterios de exclusión en la selección de las GPC, se tuvieron en cuenta los siguientes: 1) GPC para el abordaje del dolor lumbar exclusivamente en población anciana; 2) GPC en las que no se especifica el nivel de evidencia o la fuerza de las recomendaciones.

Como fuentes adicionales para comprobar la necesidad de prescripción con receta médica de los fármacos indicados en las GPC, sus interacciones con los tratamientos habituales de pacientes con HTA y/o DM y sus posibles contraindicaciones, fueron consultadas la Base de Datos del Conocimiento Sanitario del Consejo General de Colegios Oficiales de Farmacéuticos [23] (BOT plus 2.0), un tratado de Farmacología Humana [24] (Flórez, 2014) y la monografía "Nonselective NSAIDs: Adverse cardiovascular effects" [25] .

Se obtuvo en cada documento seleccionado el nivel de evidencia se- gún la escala utilizada en el mismo, bien según la clasificación del North England Evidence Based Guideline Development Project [26] o bien siguiendo el sistema GRADE (Grading of Recommendations Assessment, Development and Evaluation) [27] (tabla 1). Finalmente, teniendo en cuenta las recomendaciones sobre el abordaje farmacológico del dolor lumbar, las posibles interacciones $\mathrm{y} / \mathrm{o}$ contraindicaciones en pacientes con HTA y/o DM y el riesgo cardiovascular, se establecieron las recomendaciones específicas para los mismos. Asimismo, se construyó un algoritmo de actuación siguiendo las Buenas Prácticas en Farmacia Comunitaria en España elaboradas por el Consejo General de Colegios Oficiales de Farmacéuticos [1].

\section{Resultados}

Teniendo presentes los criterios de inclusión y exclusión, se realizó una selección de las GPC recuperadas (tabla 2). Las GPC seleccionadas [28-31] y las recomendaciones contenidas en las mismas para el abordaje farmacológico del dolor lumbar se resumen en la tabla 3. En la figura 2 se muestra el algoritmo de actuación para el tratamiento con fármacos en pacientes con HTA y/o DM que solicitan medicación 
Tabla 1 Categorización de la evidencia y fuerza de las recomendaciones

North England Evidence Based Guideline Development Project

I: ensayos clínicos controlados, metaanálisis o revisiones sistemáticas bien diseñados

II: estudios controlados no aleatorizados bien diseñados (cohortes, casos y controles)

III: estudios no controlados o de consenso
A: basadas directamente en evidencia de categoría I

B: basadas directamente en evidencia de categoría II 0 extrapoladas a partir de la evidencia de la categoria I

C: basadas directamente en evidencia de categoria III o extrapoladas a partir de la evidencia de la categoría I o II

GRADE (Grading of Recommendations Assessment, Development and Evaluation)

Calidad alta: es dificil que los resultados de nuevos estudios modifiquen la confianza en la estimación del efecto

Calidad moderada: la confianza en la estimación del efecto y su magnitud podrian cambiar con nuevos estudios

Calidad baja: es probable que nuevos estudios modifiquen la confianza en la estimación del efecto y su magnitud

Calidad muy baja: cualquier estimación del efecto es muy incierta
Fuertes: los efectos deseables de una intervención son claramente mayores que los indeseables, o viceversa

Débiles: cuando el balance entre los beneficios y riesgos es más incierto
Tabla 2 Selección de GPC y recursos basados en la evidencia

\begin{tabular}{|l|c|c|c|}
\hline \multirow{2}{*}{ Fuentes consultadas } & \multicolumn{4}{|c|}{ Dolor lumbar } \\
\cline { 2 - 4 } & DL & DE & DS \\
\hline Medline via Pubmed & 1 & 0 & 1 \\
GuiaSalud & 2 & 1 & 1 \\
National Guideline & & & \\
Clearinghouse & 4 & 3 & 1 \\
CMA Infobase & 2 & 2 & 0 \\
SIGN & 0 & 0 & 0 \\
Australia's Clinical & & & \\
Practice Guidelines & & & \\
Portal & 2 & 2 & 0 \\
Trip Database & 4 & 4 & 0 \\
NHS Evidence & 2 & 2 & 0 \\
UpToDate & 1 & 0 & 1 \\
\hline Total & 18 & 14 & 4 \\
\hline
\end{tabular}

DL: No de documentos localizados; DE: № de documentos excluidos; DS: No de documentos seleccionados.

para el dolor lumbar en la oficina de farmacia.

Las recomendaciones se refieren al manejo del dolor lumbar inespecífico e incluyen por lo general, como tratamiento de primera línea por su mejor perfil riesgo/beneficio, el uso de paracetamol en el dolor lumbar agudo o subagudo de forma aislada y de origen musculoesquelético, sin sobrepasar 3 ó $4 \mathrm{~g} /$ día. Es adecuado agregar antiinflamatorios no esteroideos (AINE) o inhibidores selectivos de la coclooxigenasa (COXIB) cuando la analgesia con paracetamol es inefectiva, no habiéndose encontrado diferencias respecto a la analgesia entre distintos AINE o COXIB y entre ambos. Estos fármacos deben ser utilizados a las dosis mínimas posibles, durante cortos periodos de tiempo (cinco a siete días) y cuando la analgesia con paracetamol ha sido inefectiva. Cuando resulte insuficiente la analgesia con estos fármacos, se sugiere combinarlos con algún opioide débil (codeína, dextropropoxifeno o tramadol) o relajante muscular no benzodiacepínico, como tizanidina, durante un período de tiempo limitado (menos de 1 ó 2 semanas).

En pacientes con o sin enfermedad cardiovascular el uso de AINE o COXIB se asocia con mayor riesgo de eventos cardiovasculares y éste depende del nivel basal de riesgo cardiovascular, del antiinflamatorio elegido y de su dosis. En pacientes sin enfermedad cardiovascular es de elección naproxeno y constituye una alternativa razonable el uso de ibuprofeno [25]. En pacientes con enfermedad cardiovascular ya establecida que requieren un AINE durante un breve periodo de tiempo o de forma intermitente también puede ser de elección naproxeno, sin embargo debe evitarse el uso de AINE cuando sea posible.

Pueden ser dispensados sin receta médica paracetamol, ácido acetilsalicílico, ibuprofeno (no todas sus presentaciones) y naproxeno (no todas sus presentaciones).

En pacientes con HTA existe interacción de paracetamol con propranolol, el cual puede ser utilizado como antihipertensivo, y de ácido acetilsalicílico con éste y otros beta-bloqueantes (atenolol, bisoprolol, carvedilol, celiprolol, labetalol, metoprolol, nebivolol y sotalol), con diuréticos (sulfonamidas: clortalidona, indapamida y xipamida; diuréticos del asa: bumetanida, furosemida, piretanida y torasemida; diuréticos ahorradores de potasio: eplerenona y espironolactona; diuréticos tiazídicos: altizida y bendroflumetiazida), con antagonistas de los receptores de la angiotensina II (ARA II) (candesartán, eprosartán, irbesartán, losartán, olmesartán medoxomilo, telmisartán y valsartán), con IECA (benazepril, captopril, delapril, enalapril, fosinopril, imidapril, lisinopril, perindopril, quinaril, ramipril y trandolapril) y con otros antihipertensivos, como aliskiren. 
Tabla 3 Recomendaciones procedentes de las GPC consultadas para el manejo farmacológico del dolor lumbar

\begin{tabular}{|c|c|c|c|c|}
\hline Autores & $\begin{array}{l}\text { Base de } \\
\text { datos }\end{array}$ & Recomendaciones & $\begin{array}{l}\text { Nivel/calidad } \\
\text { de evidencia }\end{array}$ & $\begin{array}{l}\text { Fuerza } \\
\text { de la } \\
\text { recomendación }\end{array}$ \\
\hline $\begin{array}{l}\text { Guevara et al, } \\
2011\end{array}$ & Pubmed & $\begin{array}{l}\text { A pesar de la escasa evidencia de la utilidad del paracetamol } \\
\text { en el dolor lumbar de forma aislada, puede utilizarse como } \\
\text { analgésico de primera línea en dolores crónicos y agudos de } \\
\text { origen musculoesquelético (sin sobrepasar } 3 \text { o } 4 \text { mg/dia) } \\
\text { Se sugiere agregar antiinflamatorios no esteroideos (AINE y } \\
\text { COXIB) cuando la analgesia con paracetamol es inefectiva } \\
\text { No se han encontrado diferencias respecto a la analgesia entre } \\
\text { los distintos AINE o COXIB y entre ambos } \\
\text { Deben ser utilizados a las dosis mínimas posibles, por cortos } \\
\text { periodos de tiempo (cinco a siete dias) y cuando la analgesia } \\
\text { con paracetamol ha sido ineficaz } \\
\text { Cuando resulte insuficiente la analgesia con estos fármacos } \\
\text { como monofármaco, se sugiere combinarlos con algún opioide } \\
\text { débil (codeina, dextropropoxifeno o tramadol) o relajante } \\
\text { muscular }\end{array}$ & $\begin{array}{l}\text { IV } \\
\text { IV } \\
\text { IV }\end{array}$ & $\begin{array}{l}- \\
\text { A } \\
-\end{array}$ \\
\hline $\begin{array}{l}\text { Pérez Irazusta } \\
\text { et al, } 2007\end{array}$ & GuiaSalud & $\begin{array}{l}\text { Se recomienda la utilización de paracetamol como primera } \\
\text { elección en el tratamiento de la lumbalgia aguda y subaguda } \\
\text { inespecifica por su mejor perfil riesgo/beneficio } \\
\text { Los AINE deben considerarse como fármacos de segunda } \\
\text { elección, tras el paracetamol, en el tratamiento de la lumbalgia } \\
\text { aguda y subaguda } \\
\text { La lumbalgia puede tratarse con AINE durante periodos } \\
\text { de tiempo cortos y de forma pautada. Cualquier AINE es } \\
\text { igualmente eficaz en el tratamiento de la lumbalgia } \\
\text { En pacientes con lumbalgia aguda que no responden a } \\
\text { paracetamol y AINE, pueden utilizarse opiáceos menores de } \\
\text { manera pautada } \\
\text { Si el dolor lumbar agudo persiste tras iniciar el tratamiento con } \\
\text { paracetamol o AINE a intervalos regulares, deberá valorarse } \\
\text { el asociar un miorrelajante no benzodiazepínico durante un } \\
\text { periodo corto de tiempo }\end{array}$ & $\begin{array}{l}\text { I } \\
\text { I } \\
\text { I } \\
\text { I } \\
\text { I }\end{array}$ & $\begin{array}{l}\text { B } \\
\text { D } \\
\text { B } \\
\text { D } \\
\text { D }\end{array}$ \\
\hline $\begin{array}{l}\text { Institute } \\
\text { for Clinical } \\
\text { Systems } \\
\text { Improvement } \\
\text { (ICSI), } 2012\end{array}$ & $\begin{array}{l}\text { National } \\
\text { Guidelines } \\
\text { Clearinghouse }\end{array}$ & $\begin{array}{l}\text { Se recomiendan AINE para el alivio del dolor a corto plazo en } \\
\text { pacientes con dolor lumbar agudo o subagudo } \\
\text { Los relajantes musculares pueden ser utilizados como una } \\
\text { opción en el tratamiento del dolor lumbar agudo, aunque } \\
\text { deben considerarse los efectos secundarios } \\
\text { Se recomienda el uso consciente y responsable de opioides } \\
\text { a una dosis mínima eficaz durante un periodo de tiempo } \\
\text { limitado (menos de } 1 \text { ó } 2 \text { semanas) en pacientes con dolor } \\
\text { agudo grave no controlado con paracetamol y AINE }\end{array}$ & $\begin{array}{l}\text { Evidencia } \\
\text { moderada } \\
\text { Evidencia } \\
\text { moderada } \\
\text { Evidencia baja }\end{array}$ & $\begin{array}{l}\text { Recomendación } \\
\text { débil } \\
\text { Recomendación } \\
\text { débil } \\
\text { Recomendación } \\
\text { fuerte }\end{array}$ \\
\hline $\begin{array}{l}\text { Knight et al, } \\
2016\end{array}$ & UpToDate & $\begin{array}{l}\text { Como terapia inicial, se recomienda tratamiento con AINE a } \\
\text { corto plazo (dos a cuatro semanas). Si existen contraindicacio- } \\
\text { nes para los AINE, se recomienda paracetamol } \\
\text { En pacientes con dolor refractario al tratamiento farmacoló- } \\
\text { gico inicial, se recomienda la adición de un relajante muscu- } \\
\text { lar no benzodiacepínico. En pacientes que no toleran o tienen } \\
\text { contraindicaciones a los relajantes musculares, se recomienda } \\
\text { la opción de AINE y paracetamol }\end{array}$ & ॥ & C \\
\hline
\end{tabular}




\section{Entrada en el servicio}

\section{Farmacéutico}

Obtención y evaluación de la información sobre el dolor lumbar: duración, otros síntomas, otros problemas de salud y medicamentos empleados

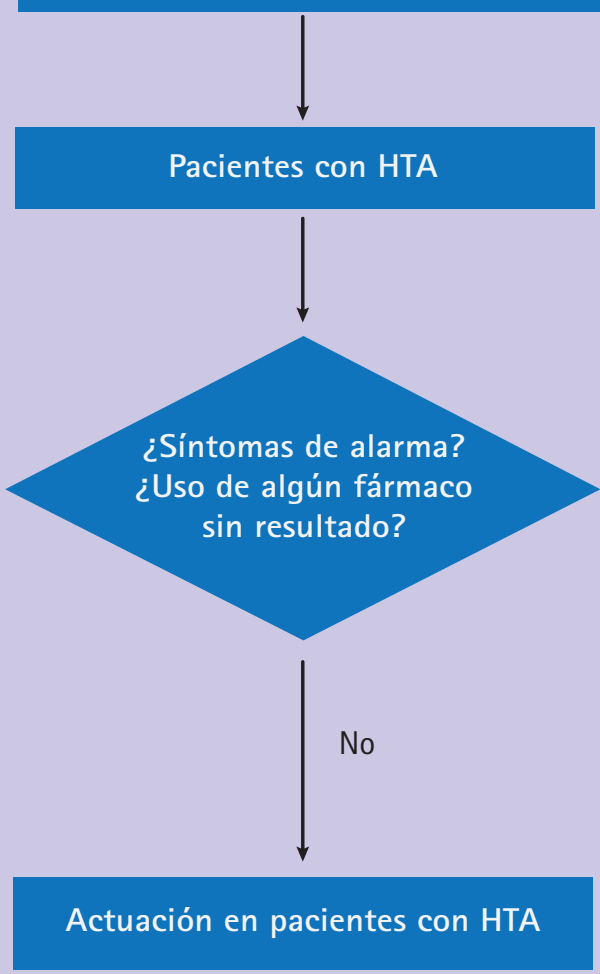

- Como tratamiento de primera línea se recomienda el uso de paracetamol en el dolor lumbar agudo o subagudo de forma aislada y de origen musculoesquelético, sin sobrepasar $4 \mathrm{~g} / \mathrm{día}$.

- Es adecuado agregar AINE cuando la analgesia con paracetamol es inefectiva. Estos fármacos deben ser utilizados a las dosis mínimas posibles, por cortos periodos de tiempo (cinco a siete días).

- Posibilidad de interacción de paracetamol con propranolol, así como de ácido acetilsalicílico, ibuprofeno o naproxeno con beta-bloqueantes, diuréticos, ARA-II, IECA y aliskiren.

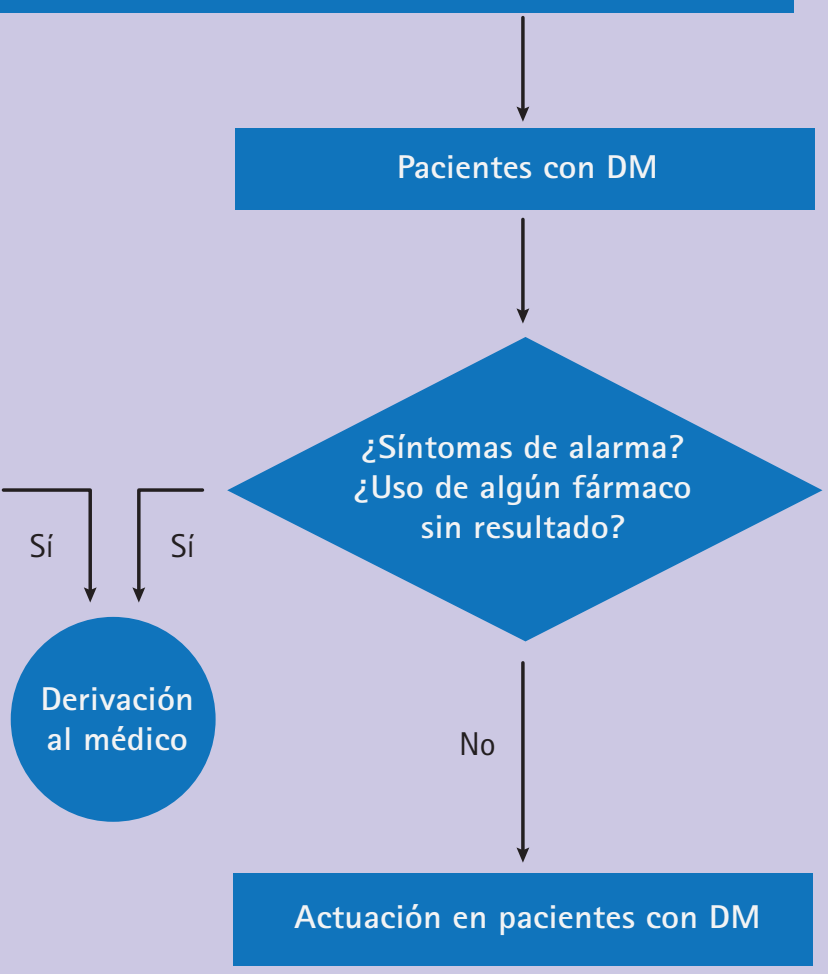

- Como tratamiento de primea línea se recomienda el uso de paracetamol en el dolor lumbar agudo o subagudo de forma aislada y de origen musculoesquelético, sin sobrepasar $4 \mathrm{~g} / \mathrm{dia}$.

- Es adecuado agregar AINE cuando la analgesia con paracetamol es inefectiva. Estos fármacos deben ser utilizados a las dosis mínimas posibles, por cortos periodos de tiempo (cinco a siete dias).

- Posibilidad de interacción de paracetamol con exenatida y lixisenatida, así como de ácido acetilsalicílico o ibuprofeno con sulfonilureas.

Figura 2 Algoritmo de actuación para el tratamiento farmacológico en pacientes con HTA y/o DM que solicitan medicación para el dolor lumbar en la oficina de farmacia 
Los efectos beneficiosos del AAS pueden ser atenuados por la administración previa o continua de algunos AINE, incluyendo ibuprofeno y naproxeno. Por lo tanto, el uso regular de AINE debe evitarse, si es posible, en pacientes que toman AAS a dosis baja para la protección cardiovascular [25].

En cuanto a ibuprofeno y naproxeno, también presentan interacción con beta-bloqueantes, ARA II, IECA, aliskiren y diuréticos.

Además, se debe tener en cuenta que los AINE pueden aumentar las cifras de presión arterial, por lo que se recomienda su monitorización en pacientes con HTA [24].

En pacientes con DM existe interacción de paracetamol con exenatida y lixisenatida, ambos utilizados como hipoglucemiantes, así como de ácido acetilsalicílico e ibuprofeno con sulfonilureas, como por ejemplo glibenclamida o clorpropamida.

Como en los casos anteriores, en estos pacientes conviene tener en cuenta la presencia como excipiente de sacarosa (en DM) o de sodio (en HTA).

Entre las medidas no farmacológicas para el tratamiento del dolor lumbar, es aconsejable hacer vida normal, evitando los movimientos o situaciones que producen dolor. Las medidas no farmacológicas constituyen la primera opción de tratamiento, sobre todo en pacientes con riesgo cardiovascular elevado porque los AINE aumentan dicho riesgo, incluso en tratamientos cortos. El reposo total no es aconsejable más allá de 1-2 días y debe tenerse en cuenta que el ejercicio mejora el dolor y la funcionalidad al compararlo con otros tratamientos conservadores [2]. Se debe evitar cargar peso y el calor local y un masaje suave pueden mejorar los síntomas.

\section{Discusión}

Mediante revisión sistemática de GPC se han formulado recomendaciones basadas en evidencia científica para el abordaje farmacológico del dolor lumbar en pacientes con HTA y/o DM. En dichas recomendaciones se han incluido sólo fármacos que pueden ser indicados en la farmacia, por no requerir prescripción médica. Asimismo, se han tenido en cuenta las posibles contraindicaciones de dichos fármacos para ambas enfermedades y se han revisado potenciales interacciones con los tratamientos antihipertensivos y/o hipoglucemiantes habituales. En los resultados se muestran las recomendaciones sobre indicación farmacéutica, así como un algoritmo de actuación para el tratamiento farmacológico en pacientes con HTA y/o DM que solicitan medicación en la oficina de farmacia.

Como posibles limitaciones de los resultados obtenidos, podría señalarse la exclusión de información adicional contenida en GPC no específicas para el síntoma seleccionado o en las que no se muestra de forma explícita el nivel de evidencia o la fuerza de las recomendaciones.

Las recomendaciones de tratamiento para el manejo del dolor lumbar inespecífico incluyen como fármaco de primera línea paracetamol, pudiendo agregarse AINE o COXIB cuando la analgesia es inefectiva. Estos fármacos deben ser utilizados a las dosis mínimas posibles y durante cortos periodos de tiempo. Las recomendaciones respecto a otros analgésicos, relajantes musculares, corticoides, antidepresivos y anticonvulsivantes como medicación coadyuvante son más variables y controvertidas, probablemente por la escasez de evidencia acerca de su efectividad [32-33].

En pacientes con HTA y/o DM deben tenerse en cuenta potenciales interacciones de paracetamol y AINE con algunos de los fármacos antihipertensivos o hipoglucemiantes utilizados habitualmente. Así, la administración de ácido acetilsalicílico, a dosis elevadas, puede dar lugar a una disminución de los efectos antihipertensivos de los beta-bloqueantes, probablemente por inhibición de la síntesis de prostaglandinas, que parecen mediar en sus efectos antihipertensivos [23,24]. Además, el ácido acetilsalicílico podría reducir ligeramente la acción de los diuréticos y, por el contrario, potenciar los efectos hipoglucemiantes de las sulfonilureas, desplazándolas de sus puntos de unión a proteínas plasmáticas. Se recomienda monitorizar la glucemia, sobre todo al iniciar y terminar un tratamiento con ácido acetilsalicílico, reajustando la posología de la sulfonilurea si fuera necesario.

También se ha podido comprobar un efecto antagonista de los AINE, a dosis elevadas, sobre los IECA, debido probablemente a la inhibición de la sintesis de prostaglandinas, que presentan efectos vasodilatadores
$[23,24]$. Por ello, se recomienda realizar un control periódico de la presión arterial. Por otra parte, los AINE podrían reducir el efecto antihipertensivo de aliskiren y precipitar el deterioro de la función renal. Especialmente en ancianos debe monitorizarse su efecto antihipertensivo y la función renal.

El dolor lumbar es un problema muy frecuente y puede ir acompañado de limitación de la movilidad. En el 90\% de los casos se debe a sobrecarga funcional o a alteraciones estructurales o degenerativas de la columna lumbar (discartrosis, escoliosis y espondilolisis, entre otros). En el $10 \%$ restante puede deberse a enfermedades inflamatorias, infecciosas o tumorales, o bien tratarse de un dolor referido [2]. La lumbalgia aguda es autolimitada y suele resolverse totalmente en unas 4-6 semanas, aunque se desarrollan síntomas crónicos en un 10-15\% de los pacientes [34-35]. Algunos medicamentos pueden causar dolor muscular, como el producido por la retirada de corticoides o el causado por amiodarona, colchicina, diuréticos, fibratos, opiodes, quinolonas, estatinas, etc. Especial vigilancia se debe tener con la aparición de dolor muscular o debilidad en pacientes tratados con fibratos y estatinas por riesgo de rabdomiolisis.

Debe ser derivado al médico el dolor lumbar que no mejora tras 2-3 semanas y constituyen indicadores de alarma el dolor producido por un traumatismo violento, el que despierta por la noche o no mejora con el reposo y el que se irradia hacia los miembros inferiores [2]. También, la existencia de sintomas acompañantes tales como fiebre, astenia, sudoración o pérdida de peso, la aparición de pérdida de fuerza o de sensibilidad en alguno de los miembros y la presencia de trastornos de esfínteres.

En conclusión, en el manejo del dolor lumbar inespecífico el fármaco de primera línea es paracetamol, pudiendo agregarse AINE cuando la analgesia es inefectiva. En pacientes con HTA existe interacción de paracetamol con propranolol y de AINE con éste y otros beta-bloqueantes, diuréticos, ARA-II, IECA y aliskiren. En pacientes con DM existe interacción de paracetamol con exenatida y lixisenatida, así como de AINE con sulfonilureas. El consumo de AINE en pacientes con HTA puede aumentar las cifras de presión arterial. 


\section{Referencias bibliográficas}

1. Grupo de Trabajo de Buenas Prácticas del Consejo General de Colegios Oficiales de Farmacéuticos. Buenas Prácticas en Farmacia Comunitaria en España. Madrid: Consejo General de Colegios Oficiales de Farmacéuticos; 2014.

2. Baos Vicente V, Faus Dáder MJ. Protocolos de Indicación Farmacéutica y Criterios de Derivación al Médico en Síntomas Menores. Madrid: Fundación Abbott; 2008.

3. Machuca M, Baena MI, Faus MJ. Guía de Indicación Farmacéutica Ind-Dáder. Grupo de Investigación en Atención Farmacéutica. Granada: Universidad de Granada; 2005.

4. Faus MJ, Amariles P, Martinez-Martínez F. Atención Farmacéutica. Conceptos, procesos y casos prácticos. Madrid: Ergón; 2007.

5. Banegas JR, Jovell A, Abarca B, Aguilar M, Aguilera L, Aranda P, et al. Hipertensión arterial y política de salud en España. Med Clin (Barc). 2009; 132(6): 222-9.

6. González P, Faure E, del Castillo A. por el Grupo de Trabajo para el Estudio del Coste de la Diabetes. Coste de la diabetes mellitus en España. Med Clin (Barc). 2006; 127(20):776-84.

7. Santschi V, Tsuyuki RT, Paradis G. Evidence for pharmacist care in the management of hypertension. $\mathrm{CPJ} /$ RPC. 2015; 148(1): 1-15.

8. Santschi V, Chiolero A, Colosimo AL, Platt RW, Tafflé P, Burnier M, et al. Improving Blood Pressure Control Through Pharmacist Interventions: A Meta-Analysis of Randomized Controlled Trials. J Am Heart Assoc. 2014; 3(2):e000718.

9. Tsuyuki RT, Maccallum L. Delivering on our potential: Pharmacists and diabetes care. CPJ/RPC. 2015; 148(1):5-8

10. Santschi V, Chiolero A, Paradis G, Colosimo AL, Burnand B. Pharmacist Interventions to Improve Cardiovascular Disease Risk Factors in Diabetes. Diabetes Care. 2012; 35(12):2706-17.

11. Gómez Ocaña JM, Revilla Pascual E, Fernández-Cuesta Valcarce MA, El-Asmar Osman A. Las 50 principales consultas en medicina de familia. Un abordaje práctico basado en la evidencia. Madrid: Agencia Laín Entralgo; 2011.

12. Morales-Olivas FJ, Estañ L. Interacciones medicamentosas. Nuevos aspectos. Med Clin (Barc). 2006; 127(7):269-75.
13. Urrutia G, Bonfill X. Declaración PRISMA: una propuesta para mejorar la publicación de revisiones sistemáticas y metaanálisis. Med Clin (Barc). 2010; 135(11):507-11.

14. Pubmed. US National Library of Medicine National Institutes of Health. USA: National Center for Biotechnology Information [acceso 12 de febrero de 2016]. Disponible en: http:// www.ncbi.nlm.nih.gov/pubmed.

15. GuíaSalud. Biblioteca de Guías de Práctica Clínica del Sistema Nacional de Salud. IACS. Gobierno de Aragón [acceso 14 de febrero de 2016]. Disponible en: http://portal.guiasalud.es/ web/guest/home.

16. National Guideline Clearinghouse. USA: Agency for Healthcare Research and Quality's (AHRQ) [acceso 15 de febrero de 2016]. Disponible en: http://www.guideline.gov/.

17. Canadian Medical Association Infobase. Clinical Practice Guidelines Database (CPGs) [acceso 15 de febrero de 2016]. Disponible en: https:// www.cma.ca/En/Pages/clinical-practice-guidelines.aspx.

18. Scottish Intercollegiate Guidelines Network (SIGN). Healthcare Improvement Scotland [acceso 15 de febrero de 2016]. Disponible en: http://www. sign.ac.uk/.

19. Australia's Clinical Practice Guidelines Portal. National Health and Medical Research Council [acceso 15 de febrero de 2016]. Disponible en: https://www.clinicalguidelines. gov.au/.

20. Trip Database [acceso 15 de febrero de 2016]. Disponible en: https://www. tripdatabase.com/.

21. National Health Service (NHS) Evidence. National Institute for Health and Care Excellence (NICE) [acceso 16 de febrero de 2016]. Disponible en: https://www.evidence.nhs.uk/.

22. UpToDate. Post TW (Ed), UpToDate, Waltham, MA [acceso 16 de febrero de 2016]. Disponible en: http://www. uptodate.com/es/home.

23. BOT Plus 2.0. Base de Datos del Conocimiento Sanitario del Consejo General de Colegios Oficiales de Farmacéuticos [acceso 16 de febrero de 2016]. Disponible en: https://botplusweb.portalfarma.com/.

24. Flórez J, Armijo JA, Mediavilla A. Farmacología Humana (6 $\left.6^{\mathrm{a}} \mathrm{Ed}\right)$. Barcelona: Elsevier; 2014.

25. Solomon DH. Nonselective NSAIDs: Adverse cardiovascular effects. En Waltham MA: UpToDate; 2016 [acce- so 16 de diciembre de 2016]. Disponible en: http://www.uptodate.com.

26. Eccles M, Clapp Z, Grimshaw J, Adams PC, Higgins B, Purves I, et al. North of England evidence based guidelines development project: methods of guideline development. BMJ. 1996; 312:760-2.

27. Gopalakrishna G, Mustafa RA, Davenport C, Scholten RJ, Hyde C, Brozek $\mathrm{J}$, et al. Applying Grading of Recommendations Assessment, Development and Evaluation (GRADE) to diagnostic tests was challenging but doable. J Clin Epidemiol. 2014; 67(7):760-8.

28. Guevara-López U, Covarrubias-Gómez A, Elías-Dib J, Reyes-Sánchez A, Rodriguez Reyna TS, Consensus Group of Practice Parameters to $\mathrm{Ma}-$ nage Low Back Pain. Practice guidelines for the management of low back pain. Cir Cir. 2011: 79(3):264-302.

29. Pérez Irazusta I, Alcorta Michelena I, Aguirre Lejarcegui G, Aristegi Racero G, Caso Martinez J, Esquisabel Martinez R, et al. Guía de Práctica Clínica sobre Lumbalgia. Vitoria-Gasteiz: Osakidetza; 2007.

30. Goertz M, Thorson D, Bonsell J, Bonte B, Campbell R, Haake B, et al. Adult acute and subacute low back pain. Bloomington (MN): Institute for Clinical Systems Improvement (ICSI); 2012 [acceso 13 de febrero de 2016]. Disponible en: http://www.guideline. gov/content.aspx?id=39319\&search=low+back+pain\#Section 420 .

31. Knight CL, Deyo RA, Staiger TO, Wipf JE. Treatment of acute low back pain. En Waltham MA: UpToDate; 2016 [acceso 16 de febrero de 2016]. Disponible en: http://www.uptodate.com.

32. Koes BW, van Tulder M, Lin CW, Macedo LG, McAuley J, Maher C. An updated overview of clinical guidelines for the management of non-specific low back pain in primary care. Eur Spine J. 2010; 19(12):2075-94.

33. Dagenais S, Tricco AC, Haldeman S. Synthesis of recommendations for the assessment and management of low back pain from recent clinical practice guidelines. Spine J. 2010; 10(6):514-29.

34. Balagué F, Mannion AF, Pellisé F, Cedraschi C. Non-specific low back pain. Lancet. 2012; 379(9814):48291.

35. Menezes Costa LC, Maher CG, Hancock MJ, McAuley JH, Herbert RD, Costa LO. The prognosis of acute and persistent low-back pain: a meta-analysis. CMAJ. 2012; 184(11):E613-24. 